\title{
Effect of adding sildenafil to clomiphene citrate versus clomiphene citrate alone on endometrium in unexplained infertility: cross-sectional, non- randomized study
}

\author{
Abstract \\ Aim of the study \\ To evaluate the endometrial thickness, pattern, and endometrial flow, in patients taking \\ clomiphene citrate plus sildenafil tablets introduced vaginally as compared to patients who \\ had no pregnancy on clomiphene citrate alone in unexplained infertility. \\ Study design: cross-sectional, non-randomized study.
}

Keywords: Estrogen, Nitric Oxide, Progesterone, Hysterosonography, Ultrasound, Clomiphene Citrate, Endometrium

Volume 7 Issue 2 - 2017

\author{
Amr Mohamed S Abdel Hamid,' Assem AM \\ Elbiaa,' Raedah A Saadoun, ${ }^{3}$ Ahmed Rateb, ${ }^{2}$ \\ Wael A Ismail Madkour' \\ 'Ain Shams University, Egypt \\ ${ }^{2} \mathrm{Al}$ Seef hospital, Kuwait
}

\begin{abstract}
Correspondence: Amr Mohamed S Abdel Hamid, Professor of Obstetrics and Gynecology, Faculty of Medicine, Ain Shams University, Cairo, Egypt, Email amoura 1000@hotmail.com
\end{abstract}

Received: May 21, 2016 | Published: May 29, 2017

\section{Introduction}

Different strategies have been adopted for inducing follicular growth and, while all systems of ovarian stimulation may improve the hormonal status of the patient, they do not necessarily lead to a receptive endometrium suitable for implantation. One of the major drugs used for infertility treatment is clomiphene citrate (a non steroidal synthetic compound with both estrogenic and anti-estrogenic activity). Clomiphene citrate stimulates ovulation by competitively inhibiting the estrogen (E) binding to the hypothalamic estrogen receptors (ER), thereby releasing the hypothalamus from the negative inhibition of endogenous (E), and leading to increase in gonadotropin pulse frequency, which consecutively induces ovulation..$^{1-6}$

Although CC is easy to use and results in ovulation in most patients (57-91\%), the pregnancy rates are disappointing (27$40 \%$ ). It is because of the adverse effects of CC mainly on quality of the cervical mucus and the endometrial development during the stimulation. Endometrial receptivity is the ability of the endometrium to successfully attach the blastocyst, to nourish it and keep it alive. This can only be achieved after the endometrium underwent a number of histological changes while also increasing in thickness. ${ }^{7-10}$ The presence of a receptive is a major factor in determining conception. A good blood supply is assumed in many studies to reflect the blood flow to the endometrium and is usually considered as an essential requirement for implantation Doppler study of uterine arteries does not reflect the actual blood flow to the endometrium. Several regimens have been proposed to improve a poor endometrial response, including treatment with Estrogens and low dose aspirin.

Nitric oxide (NO) causes vasodilatation through a c-GMP mediated pathway and NO synthetase isoforms have been identified in the uterus. Sildenafil citrate is a type 5 specific phosphodiesterase inhibitor augments the vasodilatory effects of NO by preventing the degradation of cyclic GMP. In our study, we planned to add the sildenafil to the regular clomiphene treatment in order to evaluate its effectiveness on the endometrium thickness, pattern, and vascularity, and to overcome the presumed negative clomiphene effect on the endometrial receptivity. ${ }^{11}$

Data on safety profile of Sildenafil was collected from our local erectile dysfunction clinic of the urology department where a small pilot study was performed on volunteering healthy female partners of males suffering from erectile dysfunction and both received the Sildenafil, data was collected from both partners on scoring sheets (personal communication). ${ }^{12}$

\section{Patients and methods}

The study was performed, in HMC, Abu Dhabi, Ain Shams university hospital, Cairo, Egypt and Alseef hospital Kuwait between January 2014 and January 2015. 109 Patients with unexplained infertility were approached for the study.

\section{Inclusion criteria}

I. Age: 22-35 years

II. Regular menstrual periods

III. All patients underwent full infertility workup, such as: Hormonal tests (FSH, LH, Prolactin, TSH, Estradiol, Progesterone), transvaginal ultrasound, and Semen analysis.

IV. Hysterosonography to detect the presence of endometrial focal lesions.

\section{Exclusion criteria}

1. Presence of any Organic lesion of uterus, tubes or ovaries such as endometrial polyps, ovarian cysts, leiomyomas.

2. Hormonal or semen abnormalities.

3. Non continuation of the full study time (3 cycles of treatment).

4. Presence of any medical contraindication to the medication as heart conditions. 
The study was granted the approval of the relevant medical ethical committee(s) and all recruited patients consented to the study protocol prior to starting treatment. Patients were classified into 2 groups, group A ( $\mathrm{n}=55$ patients) was prescribed clomiphene citrate (clomid $50 \mathrm{mg} \mathrm{tab}$,), one tablet every 12 hours, for 5 days from day 3 of the menstrual cycle, as well as sildenafil (Viagra, $50 \mathrm{mg}$, Pfizer Inc, New York NY-USA), vaginal tab every day for 5 days starting from day 9 of the period, While group $\mathrm{B}$ ( $\mathrm{n}=54$ patients)was prescribed clomiphene citrate (clomid, $50 \mathrm{mg}$ tab.), same dose alone in same manner. Vaginal ultrasound was performed in all subjects 3 days after the last tablet, and if no leading follicle was seen, Gonal-f (serono full data about it), 75 units subcutaneous was prescribed, once per day for 3 days, and another ultrasound was performed. Ovulation trigger was performed by HCG injection, (ovitrelle), subcutaneous, and intercourse was advised 34-36 hours and onwards after the injection. ${ }^{13}$

\section{Intervention}

A. Vaginal ultrasound to detect the endometrial thickness and pattern just before HCG trigger.

B. Doppler evaluation, to detect endometrial and sub-endometrial blood flow.

Ultrasound and endometrial zone classification was performed by Medison Accuvix machine, and was classified as follows:

After a true longitudinal view of the uterus had been obtained, the endometrial thickness was measured as the maximum thickness between the highly reflective interfaces of the endometrial-myometrial junction. The measurement included both layers of the endometrium. The surrounding low-amplitude echo layer was excluded, because it represents the inner layers of the myometrium. Endometrial morphology was classified as types A (hyper-echoic), B (iso-echoic) and $\mathrm{C}$ (triple-line) endometrium, respectively. A triple-line pattern was described as hypo-echogenic endometrium surrounded by a hyper-echogenic zone. The zones of vascular penetration into the subendometrial and endometrial regions have been defined as: Zone I i.e. the sub-endometrial zone, Zone II is the outer hyperechogenic zone and Zone III is the inner hypoechogenic zone.

The type of relative echogenicity of the endometrium and the adjacent myometrium is defined as endometrial pattern, which is usually evaluated on the day of hCG administration. Several classifications exist. The most simplified one is proposed by Sher et al. ${ }^{14}$ which consists of multilayered and non-multilayered. A multilayered endometrium has a typical triple-line pattern and reflects receptive endometrium whereas a non-multilayered pattern has homogenous hyper-echogenic or iso-echogenic endometrium compared with adjacent myometrium and was frequently associated with non-pregnant cycles.

\section{Primary outcome}

i. Endometrial thickness and pattern.

ii. Endometrial blood flow seen by Doppler

\section{Secondary outcome}

a. Pregnancy rate.

\section{Statistical analysis}

The results were tabulated and statistically analyzed using a computer program SPSS (statistic a package for social science). The sample mean (X), standard deviation (SD), and standard error of the mean were obtained for numerical variables. The Chi square test $\left(\mathrm{X}^{2}\right)$ was used in non numerical variables and paired sample $\mathrm{t}$ test was used to test the mean difference between 2 related groups. The probability $(\mathrm{P})$ value was calculated and a $\mathrm{P}$-value $<0.05$ was considered statistically significant.

\section{Results}

As it shows in Table 1, the difference between both groups is insignificant as regards age, race, as well as duration and type of infertility. Table 2 shows the endometrial thickness in both groups, it is clear that in group A (clomiphene plus sildenafil) 44 patients (80.0\%) have endometrium of 7-13 mm, which is the best thickness that favors pregnancy, whereas 32 patients $(59.2 \%)$ show similar thickness in the clomiphene group.

Table I Demographic statistics

\begin{tabular}{|c|c|c|c|}
\hline & Group $A(n=55)$ & Group $B(n=54)$ & Significance \\
\hline Mean Age (years) & $27.5(\mathrm{SD}=0.04)$ & $26.5(\mathrm{SD}=0.03)$ & NS \\
\hline $\begin{array}{l}\text { Average Duration of } \\
\text { infertility(years) }\end{array}$ & $2.5(\mathrm{SD}=0.02)$ & $1.9(\mathrm{SD}=0.0 \mathrm{I})$ & NS \\
\hline \multicolumn{4}{|l|}{ Infertility } \\
\hline Primary & 31 (56.3\%) & 29 (53.7\%) & NS \\
\hline Secondary & $24(43.7)$ & 25 (45.3\%) & \\
\hline $\begin{array}{l}\text { Race } \\
\text { Caucasians } \\
\text { (Arabs-Middle East) } \\
\text { Asians }\end{array}$ & $\begin{array}{l}47(85.4 \%) \\
8(14.6 \%)\end{array}$ & $\begin{array}{l}44(81.4 \%) \\
10(8.6 \%)\end{array}$ & NS \\
\hline
\end{tabular}

Table 2 Endometrial thickness in both groups

\begin{tabular}{llll}
\hline & Group A (n=55) & Group B (n=54) & Significance \\
\hline$<7 \mathrm{~mm}$ & $3(5.6 \%)$ & $14(25.9 \%)$ & $\mathrm{S}$ \\
$7-13 \mathrm{~mm}$ & $44(80.0 \%)$ & $32(59.2 \%)$ & $\mathrm{NS}$ \\
$>13 \mathrm{~mm}$ & $8(14.5 \%)$ & $8(14.9 \%)$ & $\mathrm{NS}$
\end{tabular}

Table 3, represents the endometrial pattern in both groups, and it shows that 52 patients $(92.7 \%)$ had a multilayered endometrium in group A, while the same pattern was present in 19 patients $(42.6 \%)$ in group B. Table 4 shows that 46 patients $(83.6 \%)$ in group A(clomiphene plus sildenafil group) demonstrated endometrial blood flow, and only 2 patients $(3.7 \%$ ) had poor flow that did not even reach the subendometrial layer, while in group B, the results were 7 patients(12.9\%) and 29 patients(53.7\%) respectively.

Table 3 Endometrial pattern in both groups

\begin{tabular}{llll}
\hline & Group A (n=55) & Group B (n=54) & Significance \\
\hline Multilayered & $52(92.7 \%)$ & $19(42.6 \%)$ & $\mathrm{S}$ \\
Non-Multilayered & $3(7.3 \%)$ & $31(57.4 \%)$ & $\mathrm{S}$ \\
\hline
\end{tabular}

Table 4 Endometrial blood flow in both groups

\begin{tabular}{llll}
\hline & Group A (n=55) & Group B (n=54) & Significance \\
\hline Zone I & $2(3.7 \%)$ & $29(53.7 \%)$ & $\mathrm{S}$ \\
Zone II & $7(12.7 \%)$ & $18(33.3 \%)$ & $\mathrm{S}$ \\
Zone III & $46(83.6 \%)$ & $7(12.9 \%)$ & $\mathrm{S}$ \\
\hline
\end{tabular}

Table 5 showed that the pregnancy rates in group A, were 13 cases $(23.6 \%)$ in total, cases were mainly in multilayered endometrium in $100 \%, 11$ patients got pregnant while showing zone III blood flow, while no pregnancy was recorded in endometrium thicker than 13 $\mathrm{mm}$, non-multilayered endometrium, and zone I endometrial blood flow. 
Table 5 Pregnancy rate in both groups

\begin{tabular}{llll}
\hline Endometrium & $\begin{array}{l}\text { Pregnancy in } \\
\text { Group A }(\mathbf{n = 5 5})\end{array}$ & $\begin{array}{l}\text { Pregnancy in } \\
\text { Group B }(\mathbf{n = 5 4})\end{array}$ & Significance \\
\hline$<7 \mathrm{~mm}$ & $\mathrm{I}$ & 0 \\
$7-13 \mathrm{~mm}$ & 10 & 3 \\
$>13 \mathrm{~mm}$ & 0 & 0 \\
Multilayered & $\mathrm{II}$ & 3 \\
Non-Multilayered & 0 & 0 & \\
Zone I & 0 & 0 & $\mathrm{~S}$ \\
Zone II & $\mathrm{I}$ & $\mathrm{I}$ & \\
Zone III & $\mathrm{I}$ & 2 & $(5.5 \%)$ \\
Total Pregnancy Rate & $\mathrm{II}(20 \%)$ &
\end{tabular}

On the other side, the total pregnancy rate in group B (clomiphene only group) was 3 cases $(5.5 \%)$, they occurred mainly in a $7-13 \mathrm{~mm}$ thickness in all cases, also in a multilayered endometrium in all cases, and 2 cases out of the 3 pregnancy cases occurred with a zone III blood flow.

\section{Discussion}

While it is widely accepted that the endometrium is a key factor for successful implantation and for establishing a healthy pregnancy, opinions are divided as to which parameter is suited best for predicting a positive outcome during cycles of assisted reproduction. We studied the effect of adding a nitric oxide enhancer to clomiphene citrate on the endometrium in patients having unexplained infertility, and undergoing controlled ovarian stimulation. Different aspects of endometrium were studied, and we tried to use simple and practical ways of evaluation of the endometrium, at last we studied the pregnancy rates in both groups. An interesting finding in the current study is that the sildenafil effect on the endometrium thickness, shown in Table 2, was mainly through its effect to avoid the presence of a (thin) endometrium, less than $7 \mathrm{~mm}$, where only 3 Patients $(5.6 \%)$ had this finding, while in the group B (clomiphene alone), the number was $14(25.9 \%)$ out of the 54 patients. This difference is statistically significant, and, by correlating it with pregnancy rates, no patients got pregnant in group B when the endometrium was less than $7 \mathrm{~mm}$ thickness, while only one patient out of the 13 who got pregnant was having a thin endometirum.

No consensus has been reached with regard to the minimum endometrial thickness required for successful pregnancy. Pregnancies did not occur when the endometrial thickness was less than $7 \mathrm{~mm} ;{ }^{15}$ however, other studies found that a minimum endometrial thickness of $6 \mathrm{~mm}$ is acceptable for implantation. ${ }^{16,17}$ Interestingly, Sundström ${ }^{14}$ reported a successful pregnancy with an endometrial thickness as little as $4 \mathrm{~mm} .{ }^{14}$ In our study, the thinnest endometrial lining for successful ongoing pregnancy was $5.8 \mathrm{~mm}$ and maximum number of conceptions occurred when the thickness was $8-10 \mathrm{~mm}$.

On the other hand, evaluation of the pattern of endometrium in the current study, shown in Table 3, showed that sildenafil effect was prominent, a fact that is represented by 52 patients out of 55 in group A $(92.7 \%)$ having multilayered endometrium, compared with 19 patients out of 54 in group B (42.6\%). Looking to the pregnancy rates, it is obvious that all of the 13 patients who got pregnant in group A fell in the category of multilayered endometrium, as well as all of the 3 patients who got pregnant in group B.

Furthermore, Table 4 represents the direct vasodilator effect of sildenafil on the endometrial blood flow, through vasodilatation of the uterine blood vessels. 46 patients $(83.6 \%)$ out of 55 showed zone
III blood flow in comparison with only 7 patients out of 54 (12.9\%), while on the other extreme, only 2 patients out of $55(3.7 \%)$ in group A had poor endometrial flow, compared to 29 patients out of 54 (53.7\%). These significant results mirror the direct effect of sildenafil on the endometrial vascularity. And by having a look on Table 5, it is clear that 11 patients out of the 13 pregnancies in group $A$ were having zone III vascularity, while 2 patients out of the 3 pregnancies in group B had zone III vascularity.

In a prospective study, Wang et al. ${ }^{18}$ studied the endometrial thickness, echo pattern and blood flow on transvaginal sonography in 182 women, eight hours prior to hCG injection. They observed a higher clinical pregnancy rate and implantation rate in women with detectable blood flow. ${ }^{18}$ The endometrial vascularity determined by three-dimensional power Doppler ultrasound was proposed to have a predictive value on the implantation rate in IVF cycles irrespective of the morphological appearance of the endometrium..$^{19}$ In another study, 3 out of 4 patients conceived after repeated implantation failure in ART, after adding sildenafil, where plasticity index decreased after 7 days of Sildenafil. ${ }^{12}$ From our study, and also from most of the previous articles, we can conclude that endometrial blood flow, as well as a normal pattern as seen by a trilaminar multilayered endometrium are essential for pregnancy to occur. They have to be evaluated together with endometrial thickness in patients undergoing ovulation induction. The role of nitric oxide effect is shown to improve the endometrial receptivity via its effect on endometrial pattern and vascularity rather than endometrial thickness. ${ }^{20-24}$

\section{Conclusion}

The use of sildenafil as an enhancer of endometrial vascularity helps in improving pregnancy rates in patients with unexplained infertility treated with clomiphene, and counteracts the poor effect of clomiphene on endometrial receptivity.

\section{Acknowledgments}

None.

\section{Conflicts of interest}

None.

\section{References}

1. Bonhoff A, Naether O, Johannisson E, et al. Morphometric characteristics of endometrial biopsies after different types of ovarian stimulation for infertility treatment. Fertil Steril. 1993;59(3):560-566.

2. Sterzik K, Dallenbach C, Schneider V, et al. In vitro fertilization: The degree of endometrial insufficiency varies with the type of ovarian stimulation. Fertil Steril. 1988;50(3):457-462.

3. Wysowski DK. Use of fertility drugs in the United States, 1973 through 1991. Fertil Steril. 1991;60(6):1096-1098.

4. Thessaloniki. Consensus on infertility treatment related to polycystic ovary syndrome. Hum Reprod. 2008;23(3):462-477.

5. Massai MR, De Ziegler D, Lesobre V, et al. Clomiphene citrate affects cervical mucus and endometrial morphology independently of the changes in plasma hormonal levels induced by multiple follicular recruitment. Fertil Steril. 1993;59(6):1179-1186.

6. Rogenhofer N, Bohlmann MK, Thaler CJ, et al. Habituelle Abortneigung: EvidenzbasierteDiagnostik und Therapie. Geburtshilfe Frauenheilkd. 2010;70(7):544-552. 
7. Navot D, Scott RT, Droesch K, et al. The window of embryo transfer and the efficiency of human conception in vitro. FertilSteril. 1991;55(1):114 118.

8. Hsu CC, Kuo HC, Wang ST, et al. Interference with uterine blood flow by clomiphene citrate in women with unexplained infertility. Obstet Gynecol. 1995;86(6):917-921.

9. Merce LT, Barco MJ, Bau S, et al. Are endometrial parameters by three-dimensional ultrasound and power Doppler angiography related to in vitro fertilization/embryo transfer outcome? Fertil Steril. 2008;89(1):111-117.

10. Ng EHY, Ho PC. Ultrasound assessment of endometrial receptivity in in vitro fertilization treatment. DSJUOG. 2010;4(2):179-188.

11. Sher G, Dodge S, Maassarani G, et al. Management of suboptimal sonographic endometrial patterns in patients undergoing invitro fertilization and embryo transfer. Hum Reprod. 1993;8(3):347-349.

12. SherG, Fisch JD. Vagina sildenafil (Viagra): a preliminary report of a novel method to improve uterine artery blood flow and endometrial development in patients undergoing IVF. Hum Reprod. 2000;15(4):806809 .

13. Singh N, Bahadur A, Mittal S, et al. Predictive value of endometrial thickness, pattern and sub-endometrial blood flows on the day of hCG by $2 \mathrm{D}$ doppler in in-vitro fertilization cycles: A prospective clinical study from a tertiary care unit. J Hum Reprod Sci. 2011;4(1):29-33.

14. Sundström P. Establishment of a successful pregnancy following invitro fertilization with an endometrial thickness of no more than $4 \mathrm{~mm}$. Hum Reprod. 1998;13(6):1550-1552.

15. Oliveira JB, Baruffi RL, Mauri AL, et al. Endometrial ultrasonography as a predictor of pregnancy in an in-vitro fertilization programme after ovarian stimulation and gonadotrophin-releasing hormone and gonadotrophins. Hum Reprod. 1997;12(11):2515-2518.
16. Gonen Y, Casper RF. Prediction of implantation by the sonographic appearance of the endometrium during controlled ovarian stimulation for in vitro fertilization (IVF). $J$ In Vitro Fert Embryo Transf. 1990;7(3):146-152.

17. Coulam CB, Bustillo M, Soenksen DM, et al. Ultrasonographic predictors of implantation after assisted reproduction. Fertil Steril. 1994;62(5):1004-1010.

18. Wang L, Qiao J, Li R, et al. Role of endometrial blood flow assessment with color Doppler energy in predicting pregnancy outcome of IVF-ET cycles. Reprod Biol Endocrinol. 2010;8:122.

19. Singh N, Bahadur A, Mittal S, et al. Predictive value of endometrial thickness, pattern and sub-endometrial blood flows on the day of hCG by $2 \mathrm{D}$ doppler in in-vitro fertilization cycles: A prospective clinical study from a tertiary care unit. J Hum Reprod Sci. 2011;4(1):29-33.

20. Sher G, Herbert C, Maassarani G, et al. Assessment of the late proliferative phase endometrium by ultrasonography in patients undergoing in-vitro fertilization and embryo transfer (IVF/ET). Hum Reprod. 1991;6(2):232-237.

21. Al-Ghamdi A, Coskun S, Al-Hassan S, et al. The correlation between endometrial thickness and outcome of in vitro fertilization and embryo transfer (IVF-ET) outcome. Reprod Biol Endocrinol. 2008;6:37.

22. Chen SL, Wu FR, Luo C, et al. Combined analysis of endometrial thickness and pattern in predicting outcome of in vitro fertilization and embryo transfer: a retrospective cohort study. Reprod Biol Endocrinol. 2010;8:30.

23. Kinay T, Tasci Y, Dilbaz S, et al. The relationship between endometrial thickness and pregnancy rates in GnRH antagonist down-regulated ICSI cycles. Gynecol Endocrinol. 2010;26(11):833-837. 